\title{
Fusing Markov Random Fields with Anatomical Knowledge and Shape Based Analysis to Segment Multiple Sclerosis White Matter Lesions in Magnetic Resonance Images of the Brain
}

\author{
Stephan Al-Zubi ${ }^{\mathrm{a}, \mathrm{c}}$, Klaus Toennies ${ }^{\mathrm{a}, \mathrm{c}}$, Nils Bodammer ${ }^{\mathrm{b}, \mathrm{d}}$, Hermann Hinrichs ${ }^{\mathrm{b}, \mathrm{e}}$ \\ ${ }^{\mathrm{a}}$ Institute for Simulation and Graphics, ${ }^{\mathrm{b}}$ Department of Neurology II \\ Otto-von-Guericke University of Magdeburg \\ ${ }^{c}\{$ stephan, klaus\}@isg.cs.uni-magdeburg.de, \\ dbodammer@neuro2.med.uni-magdeburg.de \\ ehermann.hinrichs@medizin.uni-magdeburg.de
}

\begin{abstract}
An image analysis system to segment multiple sclerosis lesions of (MR) brain volumes is proposed. The method uses Markov Random Fields (MRF) both at low and high levels. The neighborhood system used in this MRF is defined in three types: (1) Voxel to voxel: a low-level heterogeneous neighborhood used to restore noisy images. (2) Voxel to segment: a fuzzy atlas is registered elastically with the MRF then used as a-priori knowledge to correct miss-classified voxels. (3) Segment to segment: Lesion candidates are processed by a feature based classifier that looks at unary and neighborhood information to eliminate false positives.
\end{abstract}

\section{Introduction}

Multiple Sclerosis is a disease of the central nervous system. It appears as the myelin sheathes protecting nerve axons break down causing plaques. As a result $90 \%-95 \%$ of lesions occur within white matter tissue.

Segmentation based on intensity alone will not succeed because the intensity histograms of grey matter and lesions overlap in MRI images (See Fig. 1). This means that any classifier based on voxel intensity alone will classify some gray matter as lesions and lesions as gray matter.

There are a number of different approaches that employ different models for segmentation. Some researchers proposed matching a brain volume with an anatomical atlas to create grey/white mask constraints used to label lesions correctly.

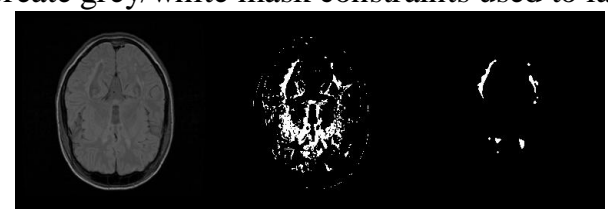

Fig. 1. Left: example of a slice showing PD contrast. Middle: lesion mask obtained by intensity based classification alone. Right: the actual lesion mask. we see how a lot of gray matter got classified as lesions. 
Warfield [1] proposes segmenting the cortex by region growing and constraining its boundary by an elastically registered anatomical atlas. This is used to make the white matter mask containing lesions and white matter that can be separated by a simple intensity based classifier. Similar work can be found for segmenting tumors in Kamber et al. [2].

Another way to classify lesions is using a feature space collected from possible candidate lesions to sort out false positives. Ardizzone [3] uses the fuzzy c-means algorithm by first obtaining a set of over-segmented regions followed by a reclustering phase. The re-clustering uses shape and intensity features to label or split unknown clusters. After this phase three masks corresponding to WM, WM+GM and $\mathrm{WM}+\mathrm{GM}+\mathrm{CSF}$ are built. The holes in these masks correspond to candidate lesions. A feature vector for each candidate is passed through a neural classifier to decide whether it's a lesion or not. Those features include: Contact with WM and GM and CSF, mean intensity, shape measures like compactness and elongation and position of the lesions like the distance from the ventricular area.

The method represented in this paper combines alignment with an anatomical model, feature based classification and image restoration using Markov Random Fields.

\section{Method}

The algorithm pipeline goes through the following steps: (1) Initial gray level segmentation. (2) Image restoration by the ICM algorithm. (3) Registration with a tissue probability distribution atlas. (4) Using the aligned atlas to correct misclassified lesion voxels. (5) Eliminating more false positive lesions by applying an MRF classifier on lesion segments. The following are the individual steps in detail:

1. Initial Grey level segmentation: The first step in the pipeline is to use a multivariate Gaussian grey level classifier where the sample mean and standard deviation are acquired by manual segmentations of some brain slices (see Fig. 1). The images are corrected for magnetic field inhomogeneities using the local intensity variations in the white matter mask.

2. Applying the ICM Algorithm to Restore Miss-classified Voxels: The segmentation is restored using all three image modalities by the Iterated Conditional modes (ICM) algorithm (for details see Besag [4]).

3. Registering Atlas with Segmented Data: In this paper the atlas $\mathbf{P}_{\mathrm{atlas}}=\left\{p_{\mathrm{i}, \mathrm{j}, \mathrm{k}}(l)\right.$ \} is a probability distribution of labels where $p_{\mathrm{i}, \mathrm{j}, \mathrm{k}}(l)$ represents the probability that the voxel i,j,k has a label $l$. B-splines are used to model the non-rigid transform [5].

4. Lesion Detection: To eliminate false positive lesions, ICM is reapplied at voxels labeled as lesions and their immediate neighbors. $\mathbf{P}_{\text {atlas }}$ is added as a new term to the energy function optimized by the ICM (i.e. $\mathrm{P}(\mathbf{X} \mid \mathbf{Y})=\mathrm{P}(\mathbf{Y} \mid \mathbf{X}) \mathrm{P}(\mathbf{X}) \mathrm{P}_{\text {atlas }}$, where $\mathbf{X}$ is the segmentation and $\mathbf{Y}$ is the image data). This has the effect of adding special information to each voxel which disambiguates between lesions and grey matter based on its probability in the atlas. Registration errors may still misclassify certain voxels therefore feature based classification is needed to improve results. 
5. Applying Shape Based MRF: False positives resulting from the previous step are further eliminated by defining individual candidate lesions segments as shape units in a Markov Random Field. Each shape unit can be reassigned a state (MS lesion, grey matter $\}$ based on shape and neighborhood features. An iterative process similar to ICM is used to re-label those units until convergence. The following is the description of the steps in detail:

(1) The watershed transform is used to isolate most individual plaques in each $\mathrm{XY}$ slice using the property that generally the intensity is bright at the center and decreases towards the edges.

(2) The shape units calculated in step (1) are considered random variables (sites) which may be relabeled as \{lesion, grey matter $\}: S=\left\{\begin{array}{lll}s_{1} & \ldots & s_{k}\end{array}\right\}, L_{S}$ : $\mathrm{S} \rightarrow\left\{1_{\text {grey_matter }}, 1_{\text {lesion }}\right\}$

(3) Unary features are computed for each shape unit that measure: compactness, elongation and distance from the center of ventricular area $\left(\phi_{\text {unary: }}\right.$ : $\mathrm{S} \rightarrow \mathbf{v}_{\text {unary }}$ ).

(4) A neighborhood system $\left(\eta_{\mathrm{S}}^{\mathrm{z}}: \mathrm{S} \rightarrow 2^{\mathrm{S}}\right)$ is defined for each shape unit where $\eta_{\mathrm{S}}{ }^{\mathrm{z}}\left(\mathrm{s}_{\mathrm{i}}\right)$ are all other shape units that have contact with $\mathrm{s}_{\mathrm{i}}$ in the $\mathrm{Z}$ direction. Similarly we define $\eta_{\mathrm{S}}{ }^{\mathrm{xy}}$ as the neighborhood that specifies contact in the XY direction.

Binary features are computed for each shape unit that includes: (a) the area of contact with gray matter (b) the area of contact with other shape units in the neighborhood system $\left\{\eta_{\mathrm{S}}^{\mathrm{z}}, \eta_{\mathrm{S}}{ }^{\mathrm{xy}}\right\}$ ( $\phi_{\text {binary }}: \mathrm{S} \rightarrow \mathbf{v}_{\text {binary }}$ ).

(5) A binary classifier $C: S \times \eta_{S}{ }^{z} \times \eta_{S}{ }^{x y} \rightarrow\left(P_{\text {lesion }}, P_{\text {grey_matter }}\right)$ is defined. It assigns higher probability that a shape unit is a lesion whenever it is more oval or contacts more other lesions or has a certain location. It assigns it a higher probability that it is grey matter when it contacts more grey matter or has a certain location.

(6) Using the neighborhood system and shape features, an iterated conditional mode algorithm is defined where each site is assigned a state of lesion or gray matter based the classifier $\mathrm{C}$. The Algorithm is iterated a few times until convergence.

\section{Results}

Experiments were conducted on three brain volumes with T1, T2, and PD weighted contrast. Each modality is of dimension $256 \times 256 \times 48$ where the voxel size is $0.97 \times 0.97 \times 3.0 \mathrm{~mm}$. Manual segmentations of the multiple-sclerosis lesions for the three volumes were obtained by a medical expert. Two measures were used to compare the manual and automatic segmentation: (1) Similarity index: The similarity index between two segments $A_{1}, A_{2}$ is a number between $[0,1]$ defined by $\mathrm{S}=2\left|\mathrm{~A}_{1} \cap \mathrm{A}_{2}\right| /\left(\left|\mathrm{A}_{1}\right|+\left|\mathrm{A}_{2}\right|\right)$. (2) Automatic and Manual segmentation volumes which show the quantity of false positives eliminated after each stage of the algorithm.

Fig. 2 shows similarity index for a typical MS patient after each stage of the algorithm. The ICM algorithm restored the image and yielded a 5\% improvement in similarity index for the whole volume. Using the atlas yields a $13 \%$ improvement in similarity. Using the shape based ICM yields a $13 \%$ improvement in similarity especially after slice -6 as shown in the figure.

Fig. 3 shows automatic versus the manual segmentation volumes for the same patient. Initially the overall correlation between manual and intensity based 
segmentation is 0.3 . The correlation became 0.87 after applying the atlas because most false positives were eliminated at this stage. After applying the shape based ICM the correlation became 0.95 as can be seen between the closely matching curves.

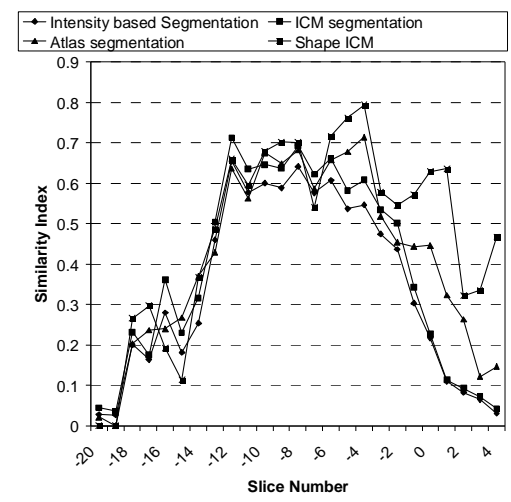

Fig. 2 Similarity Index and segmentation volumes for after each stage of the algorithm pipeline for one patient

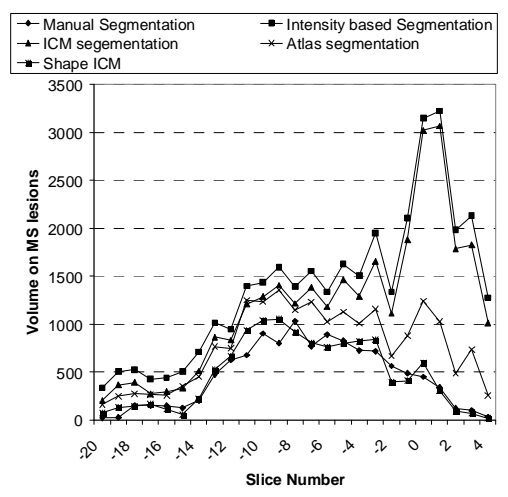

Fig. 3 Manual segmentation volume vs. Automatic segmentation volume for each stage of the algorithm.

\section{Conclusions and Future Work}

A method to segment multiple sclerosis was presented. It employs a three stage algorithm which first segments and restores the image at low level and then an anatomical atlas is used to disambiguate between lesions and gray matter. A shape based MRF in which shape units consisting of lesion slices and a neighborhood system representing contact with other lesions and gray matter is used to eliminate false positives. Future work will concentrate on refining the shape based classifier.

\section{References}

1. Warfield S, Dengler J, Zaers J, et al.: Automatic identification of grey matter structures from MRI to improve the segmentation of white matter lesions. J Image Guid Surg 1: 326-338, 1996.

2. Kamber M, Shinghal R, Collins L, et al.: Model-based 3D segmentation of multiple sclerosis lesions in magnetic resonance brain images. IEEE Trans Med Imaging 14(3): 442-453, 1995.

3. Ardizzone E, Pirrone R: An architecture for the recognition and classification of multiple sclerosis lesions in MR images. IDAMAP, 1999.

4. Besag J: On the statistical analysis of dirty pictures. J. Roy. Statist. Soc. B 48(3): 259302, 1986.

5. Rueckert D, Sonoda L, Hayes C, et al.: Nonrigid registration using free-form deformations: application to breast MR images. IEEE Trans Med Imaging 18(8): 712$721,1999$. 